\title{
Plasma membrane-localized SISWEET7a and SISWEET14 regulate sugar transport and storage in tomato fruits
}

\author{
Xinsheng Zhang ${ }^{1}$, Chaoyang Feng ${ }^{1}$, Manning Wang ${ }^{1}$, Tianlai $\mathrm{Li}^{1,2}$, Xin Liu ${ }^{1,2 \times}$ and Jing Jiang ${ }^{1,2 \bowtie}$
}

\begin{abstract}
Sugars, especially glucose and fructose, contribute to the taste and quality of tomato fruits. These compounds are translocated from the leaves to the fruits and then unloaded into the fruits by various sugar transporters at the plasma membrane. SWEETs, are sugar transporters that regulate sugar efflux independently of energy or $\mathrm{pH}$. To date, the role of SWEETs in tomato has received very little attention. In this study, we performed functional analysis of SISWEET7 $a$ and SISWEET14 to gain insight into the regulation of sugar transport and storage in tomato fruits. SISWEET7a and SISWEET14 were mainly expressed in peduncles, vascular bundles, and seeds. Both SISWEET7a and SISWEET14 are plasma membrane-localized proteins that transport fructose, glucose, and sucrose. Apart from the resulting increase in mature fruit sugar content, silencing SISWEET7a or SISWEET14 resulted in taller plants and larger fruits (in SISWEET7a-silenced lines). We also found that invertase activity and gene expression of some SISWEET members increased, which was consistent with the increased availability of sucrose and hexose in the fruits. Overall, our results demonstrate that suppressing SISWEET7a and SISWEET14 could be a potential strategy for enhancing the sugar content of tomato fruits.
\end{abstract}

\section{Introduction}

In higher plants, photosynthesis is the process by which carbon and energy and transformed into sugars in source tissues. These sugars are exported to various sink tissues and organs to support normal growth and development ${ }^{1}$. Sugars are an important nutritional component, especially in fruit crop species, and the accumulation of soluble sugars somewhat determines the quality of fruits. However, the accumulation of soluble sugars requires sugar biosynthesis, metabolism, and transport, in which specialized sugar transporters play an indispensable role ${ }^{2-4}$.

Tomato is considered an ideal model crop species because of its fleshy fruits that have important economic value $^{5}$. Sugar transport and storage are essential for

\footnotetext{
Correspondence: Xin Liu (2017500022@syau.edu.cn) or

Jing Jiang (jiangj_syau@syau.edu.cn)

${ }^{1}$ College of Horticulture, Shenyang Agricultural University, 110866 Shenyang, Liaoning, China

${ }^{2}$ Key Laboratory of Protected Horticulture of Education Ministry, 110866

Shenyang, Liaoning, China

These authors contributed equally: Xinsheng Zhang, Chaoyang Feng
}

improving tomato quality ${ }^{6,7}$. Early studies indicated that sucrose unloading in young tomato pericarps undergoing cell division occurs symplastically ${ }^{8}$. The postphloem cellular pathways in the outer pericarps are associated with switching from the symplastic route during the starch accumulation stage to the apoplastic route during the hexose accumulation stage ${ }^{9}$. This symplastic-toapoplastic switch is compatible with a facilitated transport process allowing the accumulation of soluble sugars at high concentrations without attenuating phloem unloading?.

Sucrose is unloaded from the phloem to the fruit apoplast, where it can be either directly transported into storage parenchyma cells by sucrose transporters (SUTs) through the extracellular pathway or hydrolyzed to glucose and fructose by extracellular invertase and then transported into parenchyma cells by hexose transporters ${ }^{10,11}$. Thus, the apoplastic unloading of sugar can facilitate the influx of hexoses across the plasma membranes of storage cells. In contrast, sucrose can accelerate

\section{(c) The Author(s) 2021}

(c) (i) Open Access This article is licensed under a Creative Commons Attribution 4.0 International License, which permits use, sharing, adaptation, distribution and reproduction cc) in any medium or format, as long as you give appropriate credit to the original author(s) and the source, provide a link to the Creative Commons license, and indicate if changes were made. The images or other third party material in this article are included in the article's Creative Commons license, unless indicated otherwise in a credit line to the material. If material is not included in the article's Creative Commons license and your intended use is not permitted by statutory regulation or exceeds the permitted use, you will need to obtain permission directly from the copyright holder. To view a copy of this license, visit http://creativecommons.org/licenses/by/4.0/. 
the efflux of sucrose from the phloem sink to the apoplast through sucrose concentration differences ${ }^{9}$. Three SUTs and three hexose transporters have been identified in tomato $^{12-14}$. Immunolocalization assays showed that three of these SUTs colocalized in sieve elements and that LeSUT1 and LeSUT2 are present only in the sieve tube cells of tomato fruits and not in other phloem cells or storage parenchyma cells ${ }^{12,15}$. However, three hexose transporters (SlHT1, SlHT2, and SlHT3) are expressed in the storage parenchyma cells of tomato fruits ${ }^{16}$. The cleavage of sucrose by sucrose synthase can save more energy than can cleavage by invertase in growing sink organs ${ }^{17}$. Therefore, sucrose transmembrane transport may also occur in the parenchyma cells of tomato fruits.

In tomato, the SUTs and monosaccharide transporters identified thus far are energy dependent and sensitive to the activity of proton transporters. These transporters move sucrose across the membrane by coupling with $\mathrm{H}^{+}$-ATPase in the cell membrane. Recent studies have shown that the translocation of sucrose is driven by both saturated (possibly via SUTs) and unsaturated phases, but SUTs and other known monosaccharide transporters display low levels of expression in relevant cells despite showing only saturated sucrose transport kinetics ${ }^{18,19}$. This finding indicates that there are other transporters responsible for the transmembrane transport of sugars, independent of $\mathrm{H}^{+}$-ATPase. The discovery of SWEETs (Sugars Will Eventually be Exported Transporters) has provided new insights into sugar transport mechanisms ${ }^{20}$. SWEET proteins are sugar transporters that do not require energy and are responsible for transporting monosaccharides and/or disaccharides across membranes following a concentration gradient ${ }^{21}$. Phylogenetic analysis indicated that the SWEET protein family comprises four clades, the members of which have distinct characteristics (clade I and II SWEETs prefer hexoses; clade III members mainly transport sucrose; and clade IV members are involved in the flux of fructose across the tonoplast $^{21,22}$. The multiplicity of SWEET genes in higher plants also explains their different functions in essential developmental and physiological processes, including growth and flower, pollen, nectar and seed development ${ }^{22-29}$.

Passive facilitation plays an important role in the release of sucrose from sieve tube companion cell complexes and other vascular bundle cells to fruit parenchyma cells. Among the tomato SISWEET genes, only SISWEET1a and SISWEET15 have been experimentally researched, and it has been reported that SISWEET1a is involved in sugar regulation in fruits and leaves and that SISWEET15 is responsible for fruit and seed development ${ }^{7,30,31}$. Therefore, it is necessary to explore other candidate SWEET genes involved in sustaining sugar homeostasis in tomato fruits to improve their quality. In this study, SISWEET7a and SISWEET14 were selected for further analysis based on the results of a preliminary study ${ }^{32}$. Here, the putative effects of SlSWEET7a and SISWEET14 on tomato fruits were explored.

\section{Results \\ Expression analysis of SISWEET7a and SISWEET14 during fruit development}

The results of quantitative real-time PCR analysis of 29 SISWEETs in MG Micro-Tom fruits (Fig. 1a) showed that SISWEET1e, SISWEET3, SISWEET7a, and SISWEET14 transcripts were more abundant than those of the other SISWEETs. SISWEET1e and SISWEET3 belong to clade I, whose members are involved in glucose import ${ }^{7,30,32}$, while SISWEET7a and SISWEET14 belong to clade II and clade III, respectively; the functions of these two clades have not yet been identified in tomato. Therefore, we selected SISWEET7a and SISWEET14 as genes of interest for further study.

To further explore the effects of these two genes on the development of tomato fruits, we performed an expression pattern analysis of different fruit tissues at different fruit developmental stages and (Fig. 1b, c). SISWEET7a and SISWEET14 showed similar expression patterns during fruit development. Seven days after flowering, the expression levels of both genes first peaked and then peaked again in the MG stage. However, their transcript levels decreased after the MG stage. These results suggest that these two genes might play a role during early fruit development and carbohydrate accumulation.

Subsequently, we analyzed the expression of the same two genes in different tomato fruit tissues (Fig. 1d, e). The transcript level of SISWEET7a was higher in all tissues at the MG stage than in those at the BC or RR stages and was relatively high in the peduncles and septa, especially in the vascular bundles, compared with the other tissues at the MG stage. SISWEET14 had a similar expression pattern. The transcript level of SISWEET14 was markedly higher in the peduncles, vascular bundles, septa, and, especially, placentas, at the MG stage than in other tissues and at other stages. These results indicate that SISWEET7a and SISWEET14 could play important roles in sugar accumulation from the mature to ripe stages.

To investigate the spatial expression patterns of SISWEET7a and SISWEET14, we generated transgenic plants expressing the GUS gene under the control of the SISWEET7a and SISWEET14 promoters (pSISWEET7a: GUS and pSISWEET14:GUS, respectively) (Fig. 2). The promoter activities of SISWEET7a and SISWEET14 were measured in the stamens during the later stages of flower development. A closer investigation revealed that this expression was localized in the anthers. In pSISWEET14: GUS transgenic fruits, GUS activity was detected around vascular tissues in the peduncles, sepals, and pericarps. 

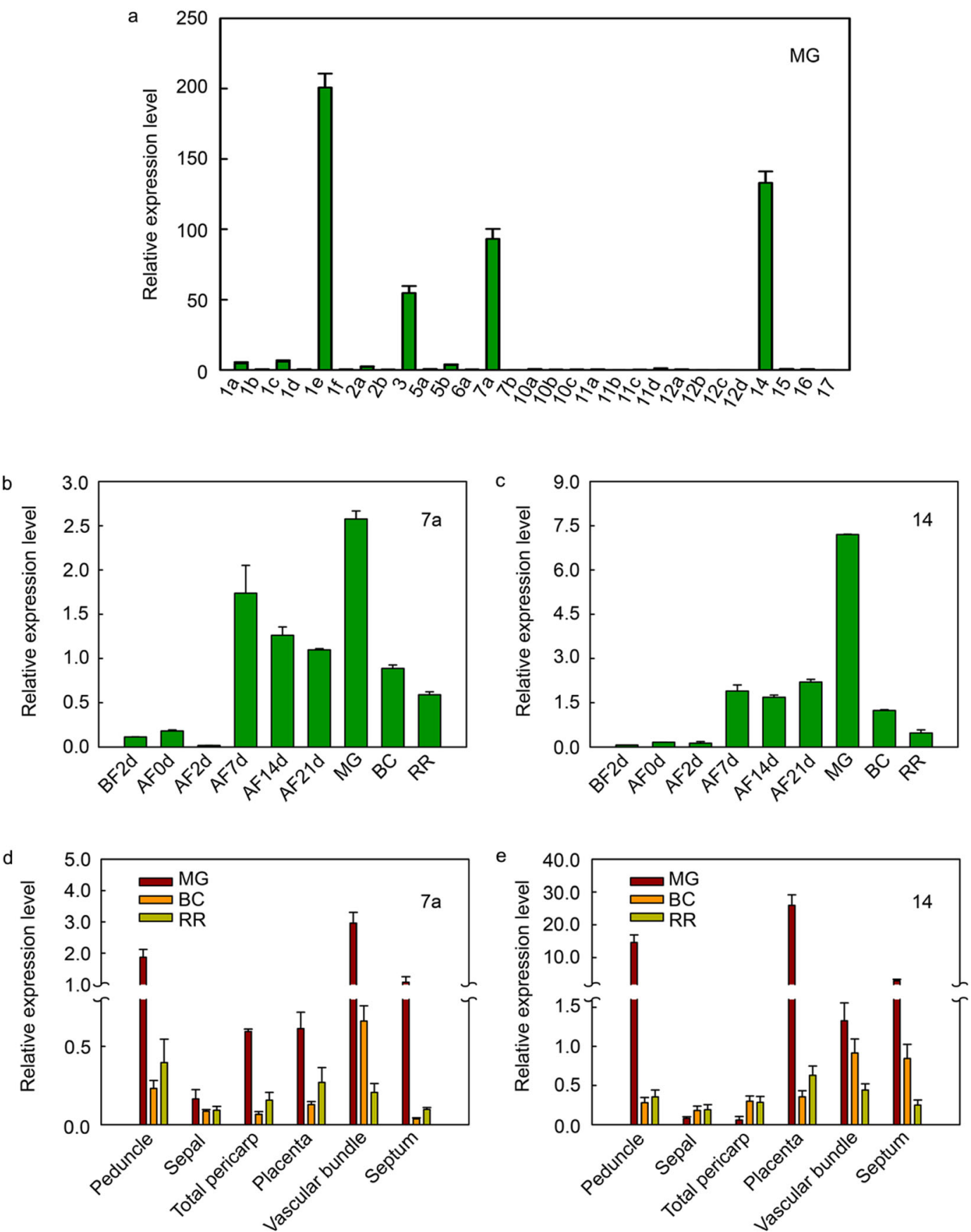

Fig. 1 Expression pattern analysis of SISWEET genes. a At the transcript level, SISWEET genes were found to exist as 29 different members in Micro-Tom mature green (MG) fruits. b, c Relative expression of SISWEET7a and SISWEET14 during fruit development in Micro-Tom. BC2d, 2 days before flowering; AFOd flowering, AF2d 2 days after flowering, AF7d 7 days after flowering, AF14d 14 days after flowering, AF21d 21 days after flowering, MG mature green, BC breaking color, RR red ripe. d, e Gene expression of SISWEET7a and SISWEET14 in different tissues during fruit development in Micro-Tom. Different tissues such as the peduncles, sepals, total pericarp (including pulp and pericarp), placentas, vascular bundles, and septa were investigated during the MG, BC, and RR stages. The data are shown as the means \pm SDs of three independently biological replicates

GUS staining was also observed in seeds at different stages. A transient GUS assay showed that the expression patterns of GUS genes driven by the promoters of the two genes were nearly identical and were mainly distributed in the vascular bundles and seeds (Supplementary Fig. 1). The expression patterns of SISWEET7a and SISWET14 showed that they may be involved in sugar accumulation and seed development. 
a

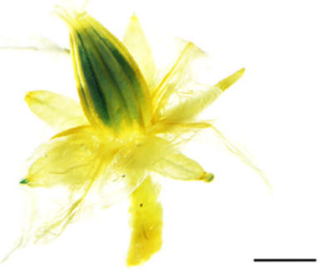

b

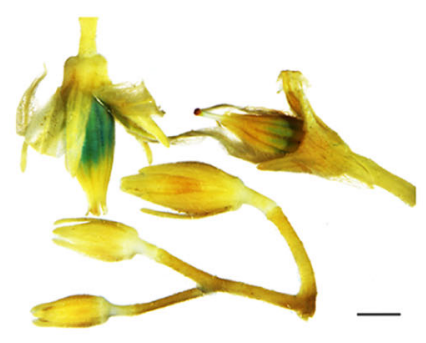

C

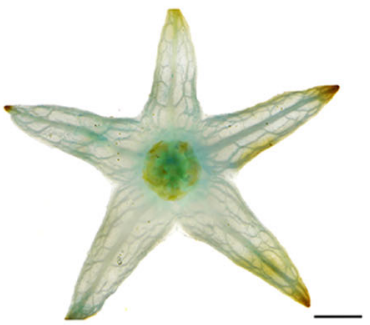

d
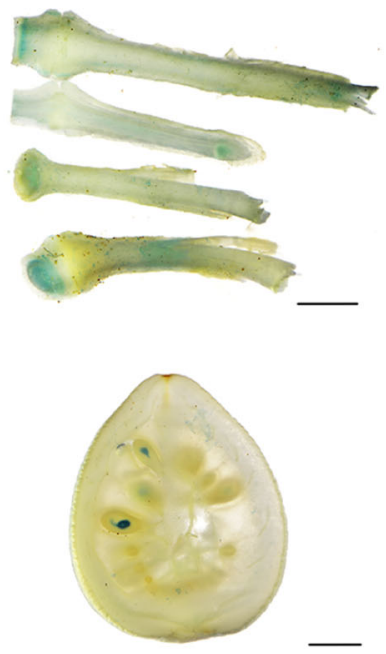

f

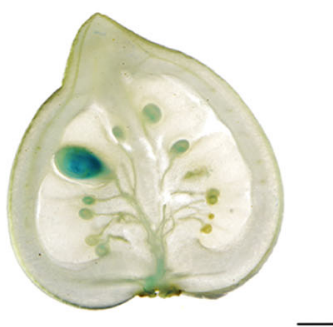

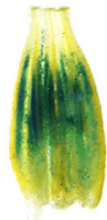
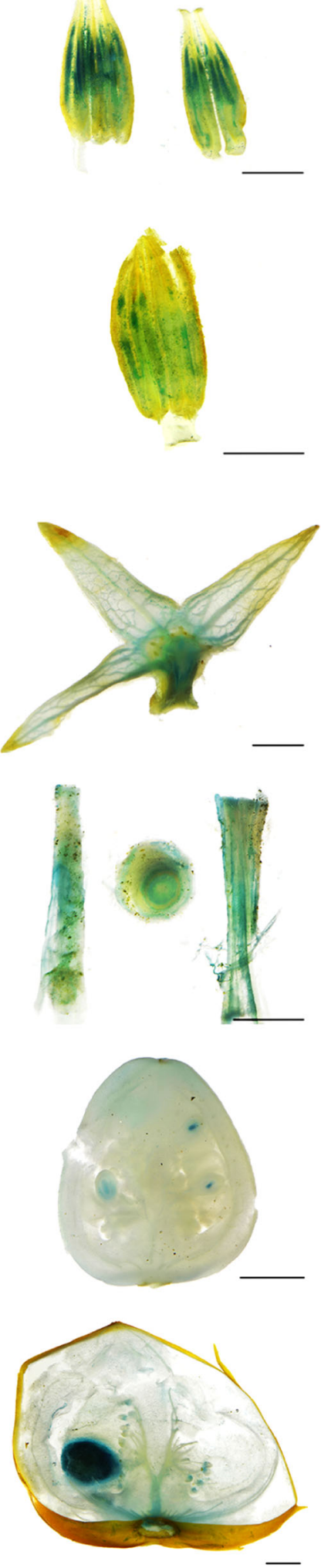

Fig. 2 Histochemical analysis of GUS activity in transgenic tomato plants. a Histochemical staining of GUS activity driven by the promoter of SISWEET7a in flowers. b-f Histochemical staining of GUS activity driven by the promoter of SISWEET14 in different tissues. b Flowers, c sepals, $\mathbf{d}$ peduncles, and $\mathbf{e}$ fruits of the expanding period and $\mathbf{f}$ fruits of the mature green stage (left) and red ripe stage (right). The scale bars correspond to $2000 \mu \mathrm{m}$ 


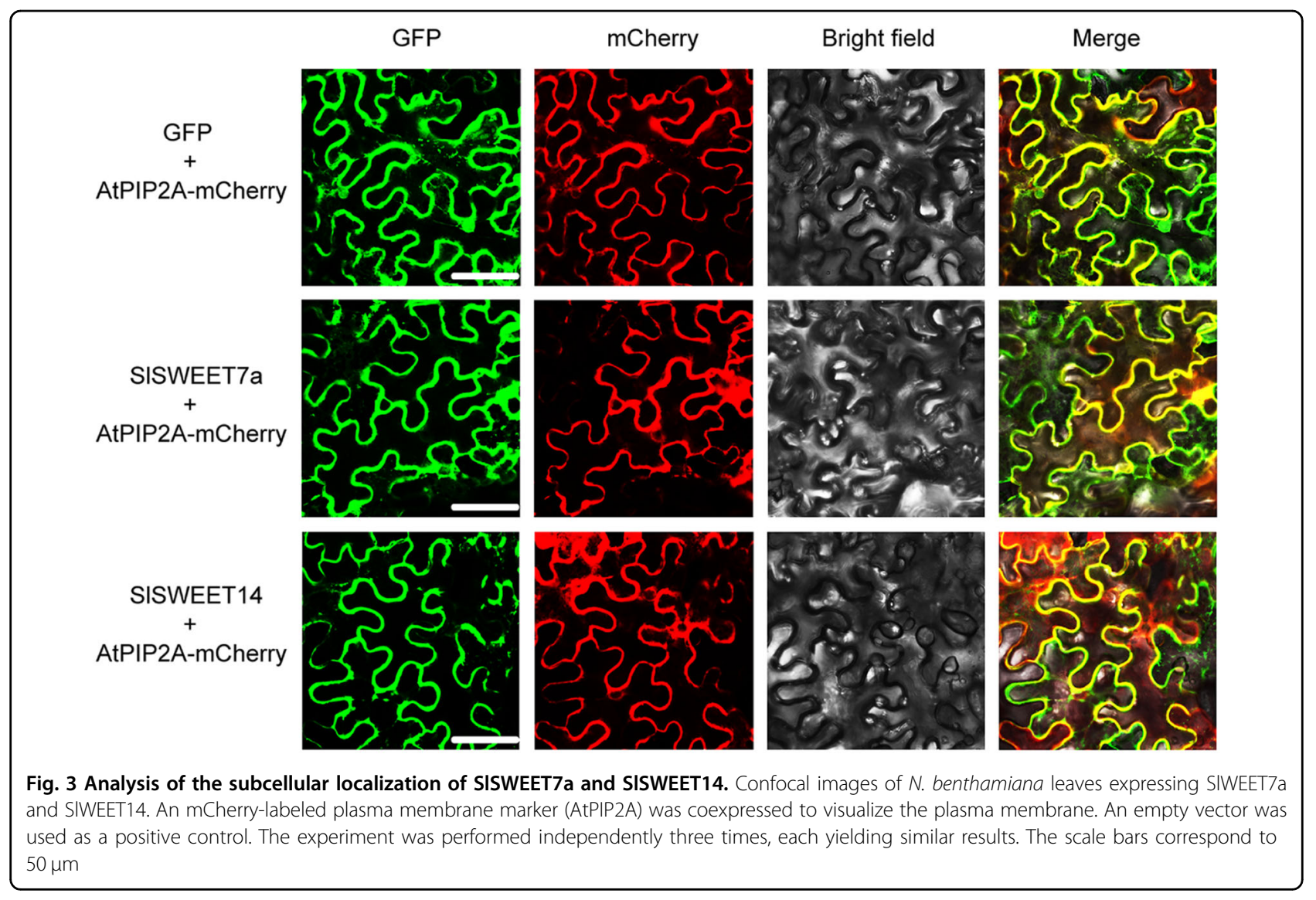

\section{Plasma membrane-localized SISWEET7a and SISWEET14 proteins}

To confirm the intracellular localization of SISWEET7a and SISWEET14, the subcellular localization of these two SWEETs was determined in Nicotiana benthamiana epidermal cells. Coexpressed pCAM35S::SISWEET7aGFP or pCAM35S::SISWEET14-GFP, together with a mCherry-labeled plasma membrane marker protein (pCAM35S::AtPIP2A-mCherry), was introduced into the epidermal cells of $N$. benthamiana leaves. A pattern of green fluorescence consistent with the predominant localization of green fluorescent protein (GFP) was observed in the plasma membrane, while additional minor fluorescence was associated within the cytoplasm. The red fluorescence of PM-mCherry overlapped with the green fluorescence of SISWEET7a and SISWEET14-GFP, indicating that SISWEET7a and SISWEET14 were mainly localized in the plasma membrane (Fig. 3).

\section{Transport substrate specificity of SISWEET7a and SISWEET14 in yeast}

The functionality of the two SISWEETs in taking up hexose and sucrose was examined via heterologous expression of their respective cDNAs in a hexose transport-deficient yeast strain (EBY.VW4000) and in the cells of a mutant SUSY7/ura3 yeast (Saccharomyces cerevisiae) strain that is deficient in the wild-type sucrose uptake mechanism (external sucrose as the sole carbon source cannot be utilized since extracellular invertase is lacking) and has sucrose synthase activity to metabolize any sucrose taken up by foreign sucrose transporters. The results showed that the expression of SISWEET7a and SISWEET14 restored the growth of EBY.VW4000 on media supplemented with glucose or fructose (Fig. 4a). Compared with their corresponding cells transformed with the control vector, the SUSY7/ura3 cells transformed with pDR195 containing each of the two SISWEET homologs (SISWEET7a and SISWEET14) and AtSUC2 (used as a positive control) exhibited faster growth on media containing sucrose as the sole source of carbon (Fig. 4b). These results indicate that SISWEET7a and SISWEET14 can transport hexose and sucrose.

\section{Identification of potential interactions between SISWEET7a and SISWEET14}

Given that other known SWEET proteins in Arabidopsis compose homo- or heterooligomeric complexes ${ }^{33}$, it is conceivable that SISWEETs function as higher-order oligomers (e.g., dimers) to perform sugar transport in tomato fruits. To confirm any interactions between the 


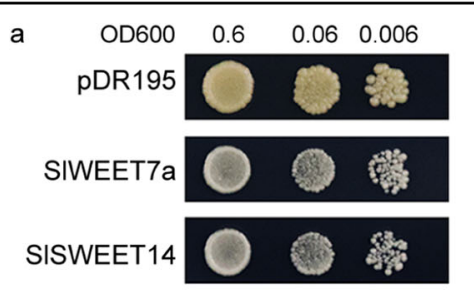

$\mathrm{SD} /$-Ura/Maltose

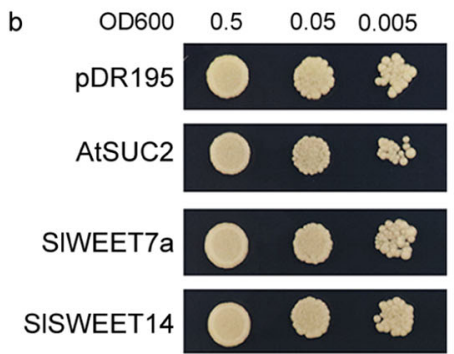

$\mathrm{SD} /$-Ura/Glucose

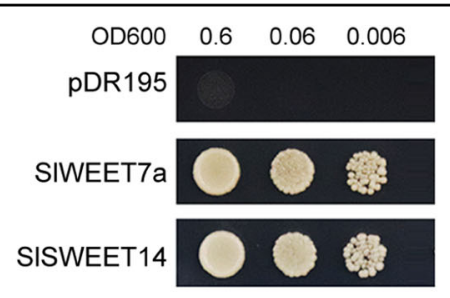

SD/-Ura/Fructose

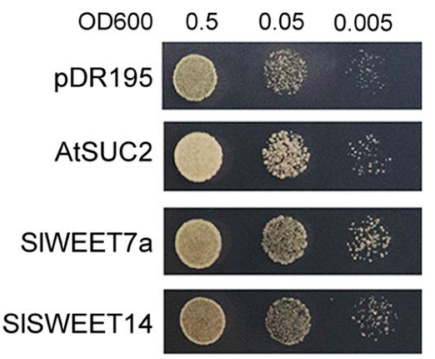

$\mathrm{SD} /$-Ura/Sucrose

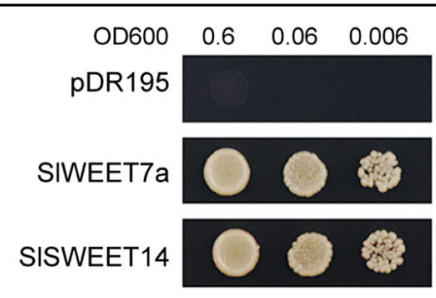

$\mathrm{SD} /$-Ura/Glucose

Fig. 4 Analysis of the transport activity of SISWEET7a and SISWEET14 in yeast cells. a Growth of the yeast mutant strain EBY.WW4000 expressing different genes in SD (-Ura) media supplemented with different carbon sources- $2 \%$ maltose, $2 \%$ fructose, or $2 \%$ glucose; yeast mutant strains transformed with the pDR195 empty vector were used as negative controls. b Growth of the yeast mutant strain SUSY7/ura3 expressing different genes in SD (-Ura) media supplemented with $2 \%$ glucose or $2 \%$ sucrose. An empty vector (pDR195) was used as a negative control, and AtSUC2 was used as a positive control. Yeast cells of strain EBY.WW4000 were grown at $30^{\circ} \mathrm{C}$ for 3 days, and yeast cells of strain SUSY7/ura3 were grown at $30^{\circ} \mathrm{C}$ for 4 days

SISWEET7a and SISWEET14 proteins, a split-ubiquitin membrane yeast two-hybrid (MYTH) system was used (Fig. 5a). The results indicate that at least SISWEET14 can form homooligomers.

To determine whether interactions can also occur in planta, oligomerization was tested for SISWEET7a and SISWEET14 using a split-GFP assay. The $\mathrm{NH}_{2}$-proximal half of YFP (nYFP) and C-proximal half of CFP (cCFP) were fused to the C-termini of SISWEET7a and SISWEET14, and the fusion proteins were transiently coexpressed in $N$. benthamiana leaves. Homooligomerization of SISWEET14 was observed in the splitGFP assay. Interestingly, SISWEET7a was found to form homooligomers and heterooligomers with SISWEET14 in planta (Fig. 5b and Supplementary Fig. 2), whereas no oligomerization was detected in the yeast two-hybrid system. All interactions seemed to occur at the plasma membrane. These split-GFP data also confirm that SISWEET7a and SISWEET14 are mainly localized in the plasma membrane.

\section{Generation of RNAi-mediated suppression lines for SISWEET7 $a$ and SISWEET14}

To suppress the specificity of SISWEET7a and SISWEET14 in tomato plants, two RNAi-mediated vectors were constructed using cDNA sequences (Supplementary
Fig. 3). In total, 7 and 15 transgenic lines were generated from the $\mathrm{T}_{1}$ generation of SISWEET7a-RNAi and SISWEET14-RNAi, respectively. To further confirm that the transcript levels of these two genes indeed decreased in the MG and RR fruits of the transgenic lines (SISWEET7ai or SISWEET14i), we performed quantitative real-time PCR analysis (Fig. 6a). We found that the expression levels of SISWEET7a and SISWEET14 significantly decreased in the silenced MG and RR fruits, respectively. Finally, three independent lines with an RNAi construct for SISWEET7a and two for SISWEET14 in the $\mathrm{T}_{4}$ generation were selected to analyze target gene suppression and for further analysis of phenotypes and sugar concentrations.

\section{Tomato sugar and starch concentrations and phenotypes were altered}

We analyzed the soluble sugar (fructose, glucose, and sucrose) and starch concentrations in the $\mathrm{T}_{4}$ generation of the RNAi-silenced SISWEET7a and SISWEET14 lines (Fig. 6b). Silencing the genes did not significantly influence the hexose concentration but significantly decreased the sucrose concentration in the mature leaves of all the transgenic lines. The starch concentrations of both SISWEET7ai and SISWEET14i were significantly higher in the mature leaves compared with other organs, where the 


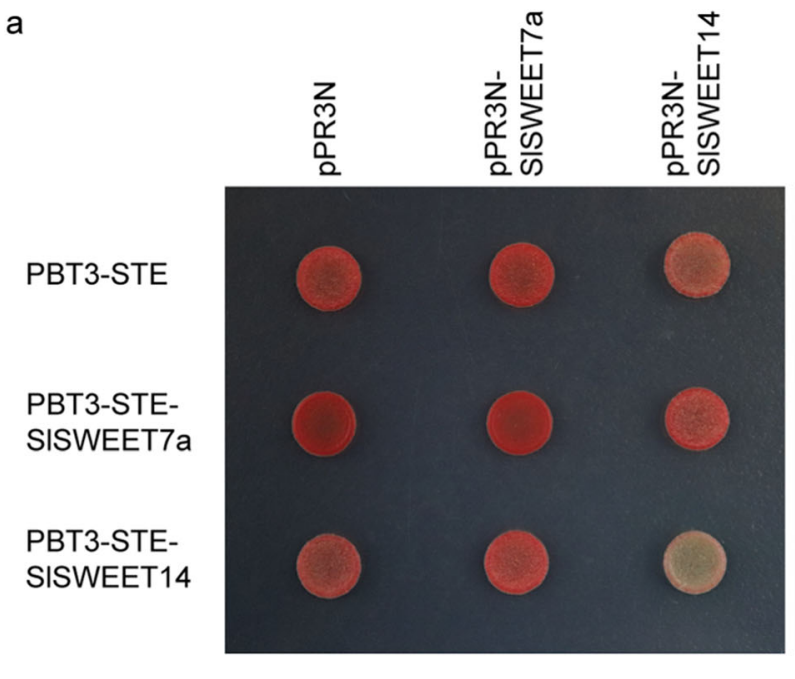

SD/-Trp/-Leu + X-gal
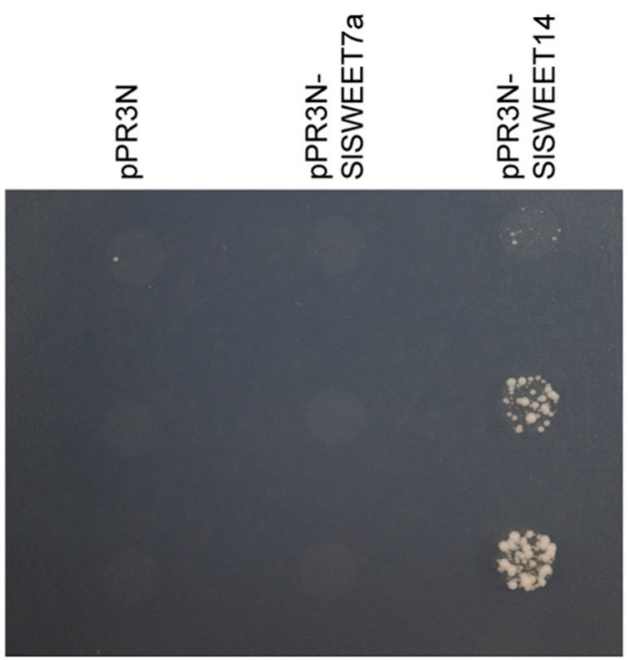

SD/-Trp/-Leu/-His/-Ade+3AT

b
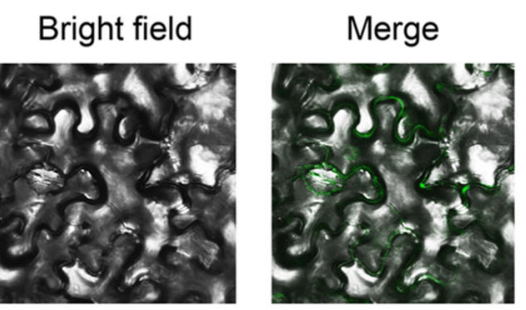

SISWEET7a-nYFP/

SISWEET7a-cCFP
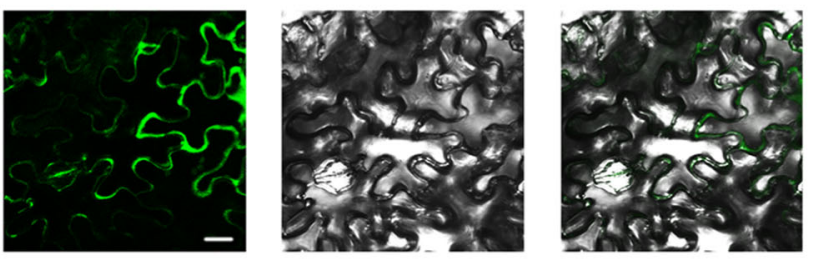

SISWEET14-nYFP/ SISWEET14-cCFP
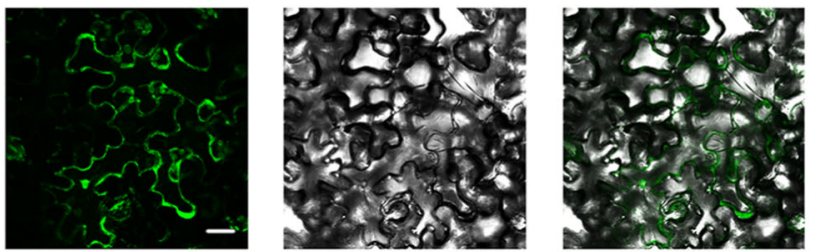

\section{SISWEET7a-nYFPI SISWEET14-cCFP}
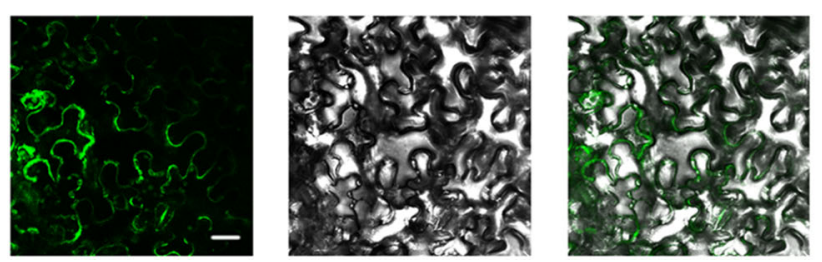

SISWEET14-nYFP/ SISWEET7a-cCFP

Fig. 5 Identification of interactions between the SISWEET7a and SISWEET14 proteins. a Interaction assays of SISWEET7a and SISWEET14 proteins via split-ubiquitin yeast two-hybrid assays. The interactions were tested using His and Ade reporter genes and 3-amino-1,2,4-triazole (10 mM) (right panel) and verified using an X-gal $(100 \mu \mathrm{g} / \mathrm{ml})$ staining assay (left panel). pR3N together with pBT3-STE, pR3N-SISWEET7a together with pBT3STE, pR3N-SISWEET14 together with pBT3-STE, pR3N together with pBT3-SISWEET7a, and pR3N together with pBT3-SISWEET14 were used as negative controls. The experiment was performed at least three times, and representative results are shown. $\mathbf{b}$ Bimolecular fluorescence complementation assays for the interaction of SISWEET7a and SISWEET14. Reconstitution of GFP-derived fluorescence and bright field and merged images are shown on the left, middle, and right, respectively. The green signal indicates GFP fluorescence. The scale bars correspond to $25 \mu \mathrm{m}$ 
a

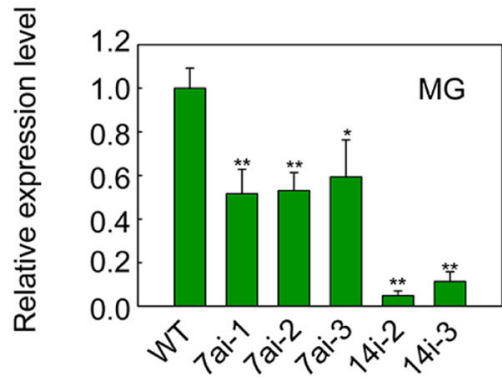

b
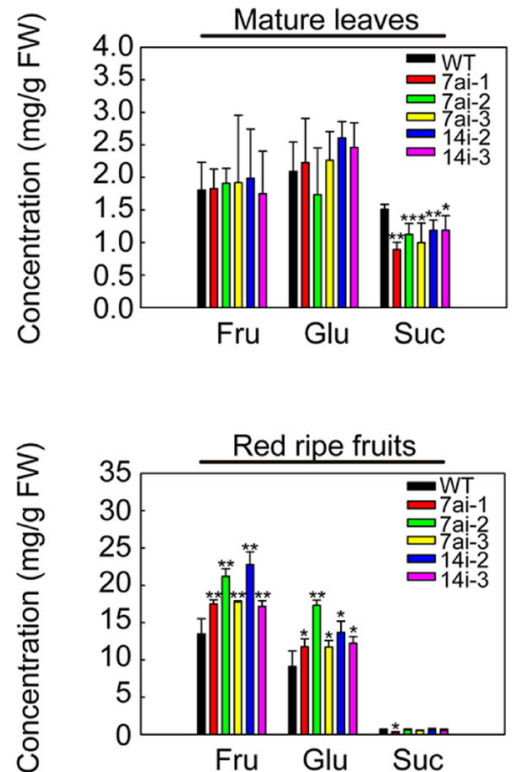
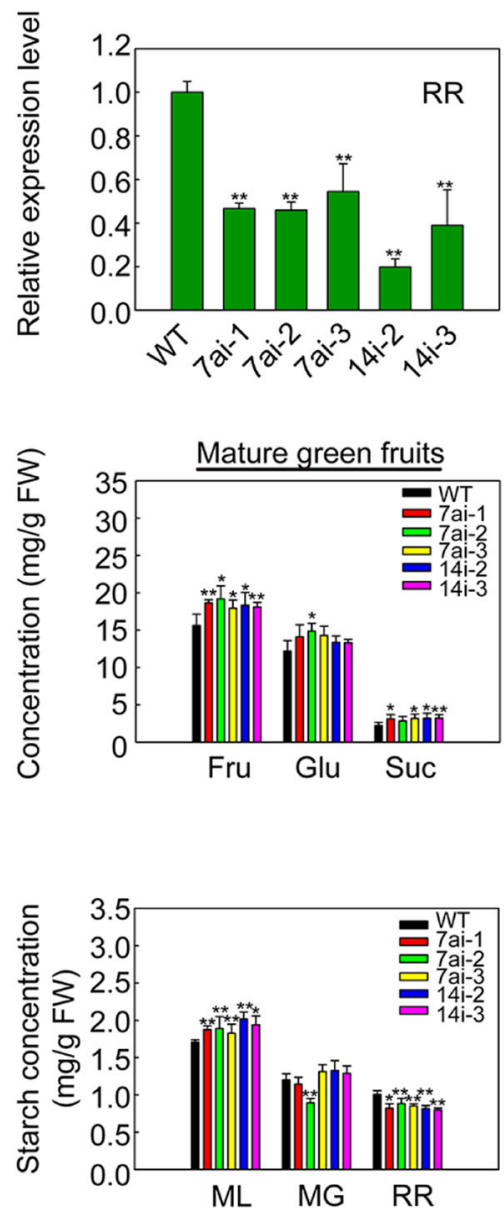

Fig. 6 Sugar concentrations in wild-type and RNAi lines. Three independent silenced lines of SISWEET7ai (SISWEET7ai-1, SISWEET7ai-2, and SISWEET7ai-3) and two lines of SISWEET14i (SISWEET14i-2 and SISWEET14i-3) were selected for experiments. a Transcript levels of SISWEET7a and SISWEET14 in SISWEET7a- and SISWEET14-silenced fruits at the mature green stage and red ripe stage, respectively. The WT (wild type) expression data were normalized to 1. The ACTIN gene was used as the internal control. b Soluble sugar concentrations (fructose, glucose, and sucrose) and starch concentrations of the wild-type and RNAi lines in mature leaves (ML), mature green fruits (MG), and red ripe fruits (RR). 7ai, RNAi lines of SISWEET7a; 14i, RNAi lines of SISWEET14. The data represent the means \pm SDs of at least five biological replicates. The asterisks indicate $P$-values $\left({ }^{*} P<0.05 ;{ }^{* *} P<\right.$ 0.01 ) according to Student's $t$ test. FW, fresh weight

concentrations increased by $\sim 7-10 \%$ in SISWEET7ai and $13 \%-18 \%$ in SISWEET14i compared with those in the WT.

In the MG fruits of all the silenced lines, significant increases in fructose concentrations and but marked differences in glucose concentrations were detected, except for the 7ai-2 lines. The fructose concentration increased by $\sim 19,22$, and $15 \%$ in the three SISWEET7ai MG fruits compared with the WT (wild type) fruits and by $\sim 17$ and $16 \%$ in the SISWEET14i fruits. The sucrose concentration also considerably increased in all the silenced lines compared with the WT, except the 7ai-2 lines, and increased by $\sim 40-44 \%$.

In RR fruit tissues, hexose (fructose and glucose) concentrations considerably increased in all the RNAi lines compared to the WT. The glucose and fructose concentrations increased by $\sim 30$ and $60 \%$, respectively, in the SISWEET7ai RR fruits compared with the WT fruits, and those of the SISWEET14i fruits increased by $\sim 60$ and $30 \%$, respectively. There were no significant differences in sucrose concentration of RR fruits among any of the RNAi lines except for the 7ai-1 line. The starch concentrations of both SISWEET7ai and SISWEET14i were significantly lower in the RR fruits than in the WT fruits. The starch concentrations in the RR fruits of the SISWEET7ai and SISWEET14i lines decreased by $\sim 12-18 \%$ and $18-20 \%$, respectively, relative to those in WT fruits.

During the growth and development of tomato plants, the height of the SISWEET7ai and SISWEET14i plants was significantly greater than that of the WT plants from the vegetative growth stage to the reproductive stage (Fig. 7a, b). The height increased by $35-48 \%$ in 
a

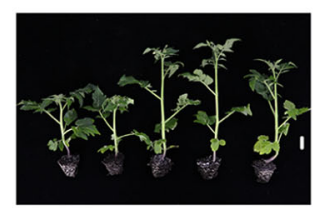
$3+x^{2}+x^{2}+v^{3}$

b

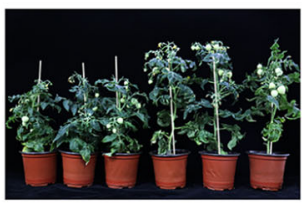
3

c

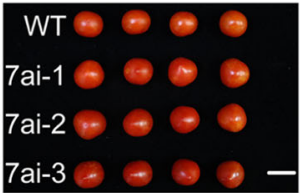

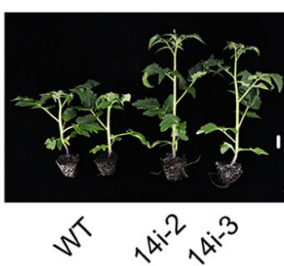
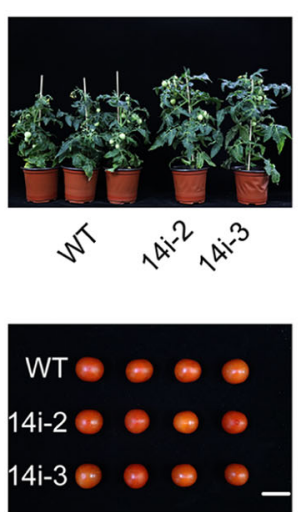

d
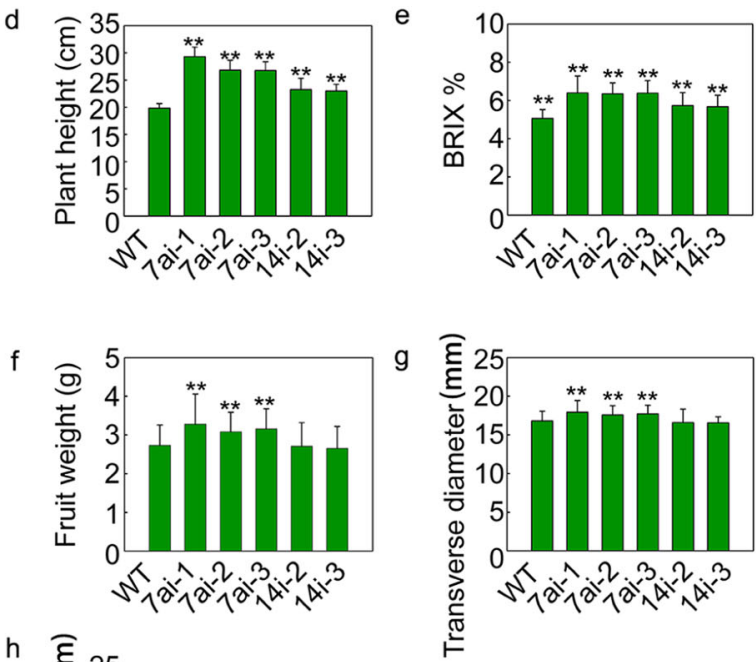

$\mathrm{h}$

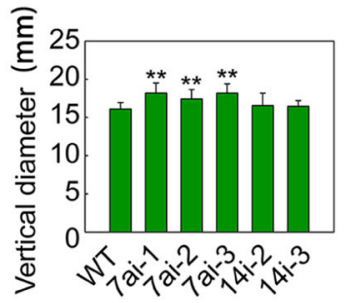

Fig. 7 Morphological phenotypes and growth parameters of RNAi lines for silencing SISWEET7a and SISWEET14. Three independent silenced lines of SISWEET7ai (SISWEET7ai-1, SISWEET7ai-2, and SISWEET7ai-3) and two independent lines of SISWEET14i (SISWEET14i-2 and SISWEET14i-3) were selected for experiments. a Plant architecture of the 7ai lines (SISWEET7ai-1 lines, SISWEET7ai-2 lines, and SISWEET7ai-3) and the 14i lines (SISWEET14i2 lines and SISWEET14i-3i lines) at 30 days after sowing the seeds; the scale bars correspond to $2 \mathrm{~cm}$. $\mathbf{b}$ Plant architecture of SISWEET7ai lines at 90 days after sowing the seeds. c Fruits from nontransgenic and transgenic lines at the red ripe fruit stage; the scale bars correspond to $2 \mathrm{~cm}$. $\mathbf{d}$ Plant height $(n \geq 12) \sim 90$ days after sowing seeds of the SISWEET7ai lines and SISWEET14i lines. e Soluble solid content (indicated by Brix \%) of fruits ( $n \geq$ $20)$ at the red ripening stage. $\mathbf{f}$ Individual-fruit weight $(n \geq 35)$. $\mathbf{g}, \mathbf{h}$ Transverse diameter and vertical diameter of fruits $(n \geq 35)$. The data represent mean values \pm SDs. The asterisks indicate $P$-values $\left({ }^{*} P<0.05 ;{ }^{*} P<0.01\right)$ according to Student's $t$ test

SISWEET7ai and by $16-17 \%$ in SISWEET14i relative to that of the WT (Fig. 7d). The soluble solid contents (Brix $\%$ also greatly increased in all SISWEET7ai and SISWEET14i lines (Fig. 7e). The individual-fruit weight and fruit diameter of the SISWEET7ai lines were notably greater than those of the WT (Fig. $7 \mathrm{f}-\mathrm{h}$ ). Indeed, the SISWEET7ai fruits were larger than the WT fruits (Fig. 7c). These results indicate that silencing SISWEET7a and SISWEET14 could result in phenotypic differences during both vegetative and reproductive growth stages.

\section{Effects of silencing SISWEET7a and SISWEET14 on genes related to sucrose metabolism or transport}

We selected genes related to sugar metabolism and transport and studied their transcription to better understand the changes in sugar levels (Fig. 8a, b). Sucrose metabolism is the process through which sucrose is degraded and resynthesized in the cytosol, vacuole, and apoplast. We analyzed the specific genes related to sugar metabolism, including 14 invertases (LIN5-9, VI, and CIN1-8), three sucrose synthases (SS1, SS2, and SS4), two sucrose phosphate synthases (SPSA2 and SPSB), four hexokinases (HK1-4), and three fructokinase genes (FK1$3)$. The expression of nearly half of the identified genes (FK1, FK2, HK2, HK3, HK4, SPSB, LIN6-9, and CIN3-5) was downregulated, while that of several other detected genes (SPSA2, LIN5, and VI) was upregulated in the SISWEET7ai MG fruits. Among them, VI was obviously upregulated by $\sim 8$-fold. The expression of these genes showed different patterns in SISWEET14i MG fruits. The transcript levels of FK1, FK3, HK4, SPSB, LIN7, and LIN9 were higher in the SISWEET14i lines compared with the other lines, while those of $F K 2, H K 3, C I N 1, C I N 3$, and CIN4 were lower. These findings suggest that SISWEET7a and SISWEET14 regulate sucrose metabolism by modulating the expression of a specific set of genes associated with sucrose degradation and synthesis.

In addition, sugar transporters play an indispensable role in the accumulation of soluble sugars. SlHT1-3 are the main contributors to hexose accumulation in tomato fruits ${ }^{16}$. The transcript levels of SlHT1 and SlHT2 were obviously downregulated in SISWEET7ai tomato MG fruits. The expression of three sucrose transporterencoding genes (SISUT1, SISUT2, and SISUT4) was also 


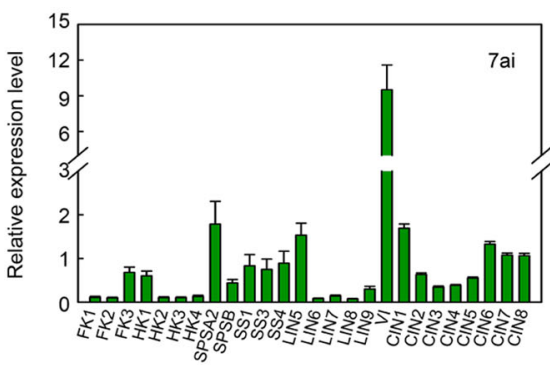

b
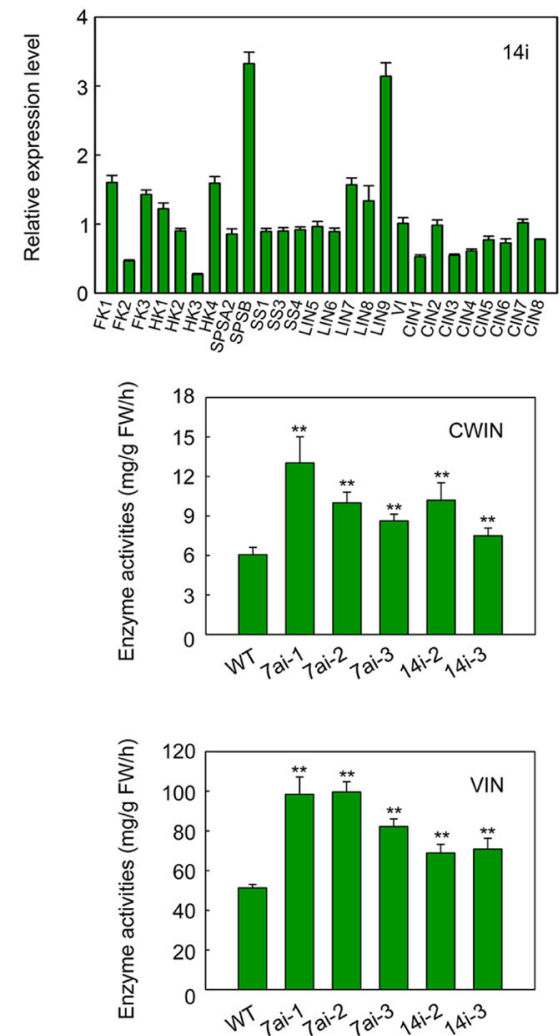
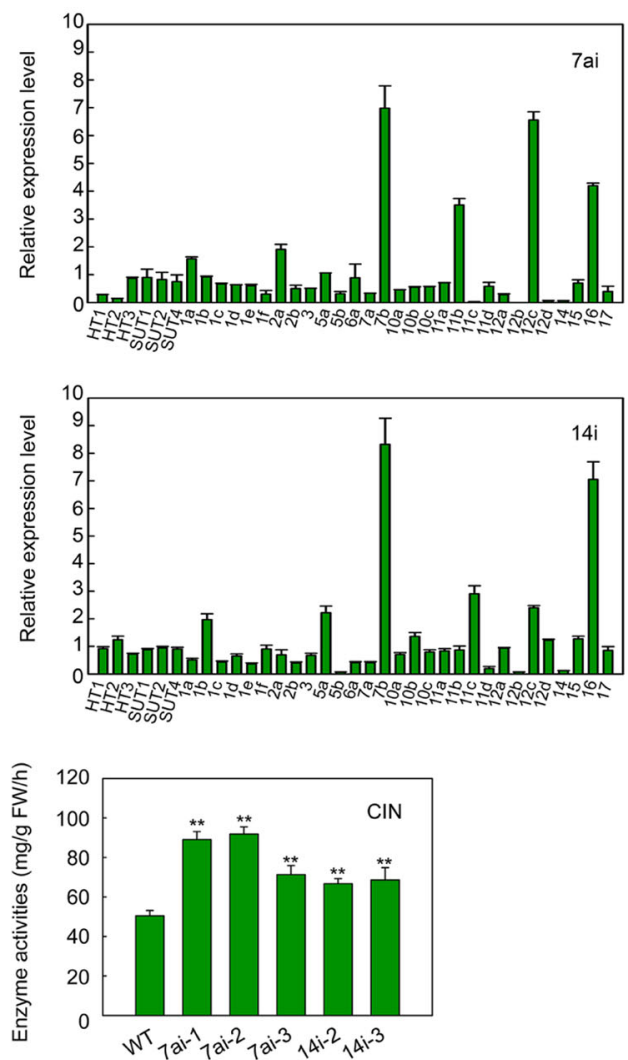

Fig. 8 Relative expression levels of genes involved in sucrose metabolism and transport as well as activities of enzymes in mature green fruits. Mixtures of cDNA from three SISWEET7ai lines or two SISWET14i lines were used for experiments. a Transcript levels of sucrose metabolism (left)- and transport (right)-related genes in SISWEET7ai (7ai) fruits at the mature green stage. $\mathbf{b}$ Transcript levels of sucrose metabolism (left)- and transport (right)-related genes in SISWEET14i (14i) fruits at the mature green stage. FK fructokinase, HK hexokinase, SPS sucrose phosphate synthase, SS sucrose synthase, LIN cell wall invertase, VI vacuolar invertase, CIN cytoplasmic invertase, HT hexose transporter, SUT sucrose transporter, 1a-17 Sugar will eventually be exported transported (SWEET). The expression data of the WT (wild type) were normalized to 1. The ACTIN gene was used as an internal control. c Enzyme activity assay of mature green fruits from silenced lines. CWIN cell wall invertase, CIN cytoplasmic invertase, VI vacuolar invertase. The data represent the means \pm SDs of at least three biological replicates. The asterisks indicate $P$-values $\left({ }^{*} P<0.05 ;{ }^{* *} P<0.01\right)$ according to Student's t test

analyzed. Among them, there were no significant changes in the expression of the three genes encoding sucrose transporters in MG fruits of SISWEET7ai or SISWEET14i tomato. The expression of all SWEET genes was analyzed in the RNAi lines. Of the clade I members encoding transporters that facilitate glucose transport, SISWEET1a and $2 a$ were highly expressed, while others were predominantly downregulated in the MG fruits of the SISWEET7ai lines. Of the potential SISWEET genes encoding sucrose transporters from clade III, SISWEET11b and SISWEET12c also significantly increased. The expression levels of SISWEET7b and SISWEET16, putative hexose-transporting SWEETs belonging to clades II and VI, respectively, were also high in the SISWEET7ai line. Among the SISWEET genes investigated, the transcript levels of SISWEET1b, $5 a, 7 b$, $11 c, 12 c$, and 16 genes significantly increased in the SISWEET14-RNAi lines. These findings suggest that the 
silencing of SISWEET7a or SISWEET14 also influences the expression of other members of the SWEET family.

\section{Effects of silencing SISWEET7a and SISWEET14 on invertase activity}

To better understand why the hexose concentration changed in the SISWEET7a and SISWEET14 RNAi lines, invertase enzyme activities were analyzed. As shown in Fig. 8c, the activity of three invertase enzymes (cell wall invertase, cytoplasmic invertase, and vacuolar invertase) significantly increased in comparison to that in the WT. Cell wall invertase activity increased by $42-115 \%$ in the SISWEET7ai line and by $23-68 \%$ in the SISWEET14i line; cytoplasmic invertase activity increased by $41-82 \%$ in the SISWEET7ai lines and 32-35\% in the SISWEET14i line; and vacuolar invertase activity increased by $60-94 \%$ in the SISWEET7ai line and $34-38 \%$ in the SISWEET14i line. These results indicated that silencing SISWEET7a and SISWEET14 could improve invertase activity and promote sucrose cleavage in the intracellular and extracellular space.

\section{Discussion}

Plasma membrane-localized SWEET7a and SISWEET14 in tomato could transport hexoses and sucrose

Previous studies have shown that most SWEET proteins are localized on the plasma membrane and may be the primary site at which sugar flux is regulated ${ }^{7,30}$. SWEET proteins, which have been identified as bidirectional transporters, can regulate both cellular uptake and efflux of sugars, but there are differences in substrate specificity between the different SWEETs ${ }^{22,34}$. Phylogenetic analysis has indicated that SISWEET7a and SISWEET14 belong to clades II and III, respectively ${ }^{30}$. Our results showed that these two SWEETs are located on the plasma membrane and are capable of transporting hexose (glucose and fructose) and sucrose, similarly to clade III members AtSWEET11 and AtSWEET12 ${ }^{35}$. In addition, VvSWEET10 from clade III is a hexose-affinity transporter in grape ${ }^{36}$. Accordingly, the substrate specificity of SWEETs may also be dependent on species, not merely on SWEET clade. SWEET proteins have a broad range of substrate transport functions and can transport not only sugars but also phytohormones (gibberellins) and other substrates ${ }^{3,37}$.

SWEETs assemble into oligomers to establish translocation pores to transport sugars, especially for larger substrates such as sucrose or fructose ${ }^{34,38}$. Coimmunoprecipitation experiments have suggested that both OsSWEET2b and AtSWEET1 can form homooligomers ${ }^{39}$. Split-ubiquitin yeast two-hybrid and split-GFP assays have also demonstrated homo- and heterooligomerization of SWEETs in Arabidopsis thaliana ${ }^{33,39}$. We detected similar interactions between SISWEET7a and SISWEET14.
The split-ubiquitin system and split-GFP system showed that SISWEET14 can form homooligomers. The split-GFP system indicated that SISWEET7a and SISWEET14 can form heterooligomers, but the split-ubiquitin system did not. Similarly, AtSWEET6 homooligomerization was observed only in the split-GFP assay, and AtSWEET4 could form homooligomers in a split-GFP system; however, this was not observed in a yeast two-hybrid system ${ }^{33}$. Therefore, whether the oligomerization of SISWEET7a and SISWEET14 plays an important role in transporting hexoses across the cell membranes of tomato fruits requires further investigation.

\section{SISWEET7a and SISWEET14 mediate sugar transport and metabolism}

There are an increasing number of reports concerning SWEET functions in sink organs, especially fleshy fruits. In Ananas comosus, 12 of the 21 AnmSWEETs were shown to be expressed in different stages of fruit development and ripening. AnmSWEET5 and AnmSWEET11 are especially highly expressed in the early stages of fruit development ${ }^{40}$. In apple, MdSWEET9b and MdSWEET15a likely regulate fruit sugar accumulation ${ }^{41}$. Overexpressing VvSWEET10 improved hexose accumulation in grapevine calli and tomato fruits ${ }^{36}$. In tomato, overexpressing plasma membrane-localized SISWEET1a promoted glucose efflux from fruits, resulting in an altered hexose composition in ripening tomato fruits ${ }^{7}$. Interestingly, hexose accumulation also increased in SISWEET7a- and SISWEET14-silenced tomato fruits in the present study. The physiological roles of the two genes in tomato fruits may also involve hexose efflux. Our findings showed that SISWEET7a and SISWEET14 were expressed at the highest levels at the MG stage. SISWEET7a was expressed specifically in the peduncle and vascular bundle tissues, and SISWEET14 was expressed specifically in the peduncles and especially in the placenta, vascular bundles, and seeds. In the placental phloem parenchyma of tomato fruits, where sucrose is unloaded via an apoplastic pathway, the phloem parenchyma located on the border between the placenta and the seed is a possible site for the apoplastic unloading of sucrose $^{42}$.

Unloaded sucrose can be either directly transported into fruit parenchyma cells by SUTs or hydrolyzed into fructose and glucose by cell wall invertase (CWIN) in the apoplast and then transported into parenchyma cells by hexose transporters (HTs) ${ }^{1}$. Excessive amounts of hexose inhibit the growth of reproductive tissues such as pollen tubes $^{43}$. The coexpression of SlHTs and SISWEETs may be required to sustain cytosolic sugar homeostasis ${ }^{44}$. In this study, we observed that hexose concentrations increased in the MG fruits or RR fruits of the silenced lines. As expected, cell wall invertase activities increased in 
response to silencing SISWEET7a and SISWEET14. In addition, cytoplasmic invertase and vacuolar invertase activities increased and could promote the hydrolysis of sucrose in the MG fruits of the SISWEET7ai and SISWEET14i lines. The higher amounts of hexose that accumulated in the SISWEET7ai and SISWEET14i lines may be attributed to the elevated invertase activities. Interestingly, sucrose concentrations increased in the MG fruits of the SISWEET7ai and SISWEET14i lines. There were no drastic differences in the expression of SISUT genes between the wild-type and transgenic fruits, but the transcript levels of SIHT1 and SlHT2 decreased in the SISWEET7ai lines. SWEETs may facilitate sucrose or hexose transport across membranes, as previous reports have also shown functional redundancy among SWEET family members ${ }^{45}$. Thus, in addition to SlHTs and SISUTs, some SWEETs may facilitate hexose and sucrose transport. Among the SISWEETs investigated, the expression of $7 b, 11$ (11b or $11 c), 12 c$, and 16 was upregulated in both RNAi lines. VvSWEET7 is a mono- and disaccharide transporter in grape $^{46}$; moreover, AtSWEET16, a bidirectional vacuolar sugar facilitator, plays key roles in maintaining sugar homeostasis, and overexpression of AtSWEET16 results in a decreased fructose contents in leaves ${ }^{47}$. Compounds $7 b$ and 16 could also play important roles in regulating sugar homeostasis. AtSWEET11 and AtSWEET12 from clade III were identified as key players in sucrose efflux from phloem parenchyma cells for phloem loading, and the sucrose content increased in the atsweeet11;12 double mutant ${ }^{20}$. In the present study, suppression of SISWEET7a and SISWEET14 resulted in a sucrose concentration decrease in mature leaves but an increase in MG fruit sucrose concentrations in the SISWEET7ai and SISWEET14i lines. This indicated that suppression of SISWEET7a and SISWEET14 could promote increased sucrose allocation from the leaves to MG fruits; compounds 11 and $12 c$ may play key roles in this process. Accordingly, SISWEET7a and SISWEET14 may be important limiting factors regulating sucrose unloading in tomato fruits and may play an important role in maintaining cytosolic sugar homeostasis.

In addition, $F K$ and $H K$ are involved in hexose metabolism, hexose sensing, and signaling by phosphorylating hexose $^{48,49}$. The transcript levels of $F K$ and $H K$ showed different patterns in the MG fruits of the SISWEET7ai and SISWEET14i lines. SPS overexpression increases sucrose turnover and the amount of sucrose unloaded in fruits ${ }^{9,50}$. Although two different SPS-encoded genes were induced by silencing SISWEET7a and SISWEET14, we nevertheless observed increased sucrose concentrations in the MG fruits of the SISWEET7ai and SISWEET14i lines. Furthermore, CWIN, encoded by the LIN gene, can influence the sugar composition in fruits, and the increase in CWIN activity by silencing its inhibitor was shown to increase fruit hexose levels ${ }^{42}$. LIN5 was upregulated in the SISWEET7ai line, and LIN7, LIN8, and LIN9 were induced in the SISWEET14 line. Cytoplasmic invertase (CIN), encoded by CIN, and vacuolar invertase (VI), encoded by $V I$, can regulate the sucrose/hexose ratio and lead to increased hexose levels and decreased cytosolic sucrose levels ${ }^{2,51}$. Moreover, CIN1 was induced in the SISWEET7ai line, and $V I$ was significantly upregulated. These results showed that silencing of SISWEET7a and SISWEET14 could influence sugar metabolism. Overall, SISWEET7a and SISWEET14 are two possible key players in sucrose unloading and sugar metabolism in tomato fruits.

\section{SISWEET7a and SISWEET14 could be regulated by phytohormones or sugars and could affect the growth and development of tomato}

Sugars are not only important carbon and energy sources and structural constituents of cells but also essential signaling molecules ${ }^{52}$. The expression of various genes in plants is affected by sugars. The activity of SWEETs can also be feedback regulated by their substrates $^{21,53}$. Our findings demonstrate that SlSWEET7a and SISWEET14 could respond to sugars (Supplementary Fig. 4). In addition, sugar signaling and the phytohormone pathway may exhibit crosstalk to modulate critical growth processes such as embryo establishment, seed germination, and seedling and tuber growth ${ }^{49,54}$. Notably, many sugar-responsive and hormone-responsive elements were found to be present in the promoters of SISWEET7a and SISWEET14 (Supplementary Table S4, Supplementary Table S5). These phytohormones may thus have an important role in their regulation, as SISWEET7a and SISWEET14 could respond to phytohormones (Supplementary Fig. 4). These results show that SISWEET7a and SISWEET14 might be involved in biological activities regulated by different phytohormones or sugars.

We found that the transgenic fruits with silenced SISWEET7a were larger than the WT fruits. Silencing SISWEET7a improved hexose accumulation. Moreover, altered sugar signaling may promote cell expansion and generate larger fruits. In addition, vacuolar invertase (VI) could promote cell expansion through crosstalk between sugar signaling and cell wall-associated kinases or phytohormones ${ }^{1}$. Therefore, the larger size of fruits of the SISWEET7a-silenced lines may also be attributed to the elevated expression of VI. Similar results have been obtained when the apple hexose transporter MdHT2.2 is overexpressed-increased hexose concentrations and fruit sizes $^{55}$. SISWEET7a and SISWEET14 probably have multiple physiological functions depending on their tissue localization. As reported in previous studies, overexpression of SISWEET1a in tomato tissues decreased the 
hexose content in the fruits, virus-induced gene silencing of SISWEET1a reduced hexose accumulation in young leaves ${ }^{7,30}$, and loss of SISWEET15 function inhibited fruit and seed development ${ }^{31}$. It has also been reported that plant height can be affected by altered expression of $S W E E T$ genes. For example, rice plants were shorter than WT rice plants when OSSWEET14 was overexpressed ${ }^{56}$. In the present study, we also observed significantly increased height of the silenced lines. SISWEET7a and SISWEET14 may also regulate plant growth and development via crosstalk among signaling pathways. As reported previously, FT-like protein (StSP6A) interacts with StSWEET11 to mediate source-sink regulation and affect tuber development via photoperiodic and sucrose signaling pathways ${ }^{24}$. However, the mechanism by which SISWEET7a and SISWEET14 regulate the growth and development of tomato needs further clarification.

In the present study, we identified two SISWEET genes, namely, SISWEET7a and SISWEET14, that are capable of transporting hexose and sucrose molecules across the plasma membrane. These two genes, which are mainly expressed in the fruits, could be directly or indirectly involved in sucrose unloading in tomato fruits. In addition, silencing SISWEET7a and SISWEET14 affected hexose accumulation in tomato fruits and influenced vegetative and reproductive growth.

\section{Materials and methods}

\section{Plant material and growth conditions}

Tomato (Solanum lycopersicum L. 'Micro-Tom') seeds were used for genetic transformation in our study. Both wild-type tomato plants and transgenic tomato plants were grown in a greenhouse with a $16 \mathrm{~h} / 8 \mathrm{~h}$ (light/dark) photoperiod at $25^{\circ} \mathrm{C}$ and a relative humidity of $50-70 \%$. Anthesis dates were determined according to the number of days after the sepals were removed. Wild-type tomato fruits were collected 2 days before anthesis and $0,2,4,7$, 14, and 21 days after anthesis and at the mature green (MG), breaking color (BC), and red ripe (RR) stages for expression analysis. In addition, different tissues from at least three plants, including the peduncle, sepal, total pericarp, placenta, vascular bundle, and septum tissues, were sampled at the MG, BC, and RR stages for expression analysis. Transgenic tomato fruits and leaves from at least five plants were collected at the mature green (MG) and red ripe (RR) stages to measure sugar and starch contents, and at least two fruits of each plant at each stage were sampled and pooled together. At each sampling point, at least three fruits (except for the transgenic lines) or three mature leaves (from the same position on each plant) from at least three plants were randomly selected and pooled together, and samples from each plant constitute one biological replicate. All the samples were immediately frozen in liquid nitrogen and kept at $-80^{\circ} \mathrm{C}$ until analysis.

\section{RNA isolation and quantitative real-time PCR}

Total RNA was extracted from the different samples using a Simple Total RNA Kit (Tiangen, Beijing, China). cDNA was synthesized for use in quantitative real-time PCR assays as described previously ${ }^{32}$. Data analysis was carried out using Bio-Rad CFX Manager Software, following the $2^{-\triangle \Delta C T}$ method. The tomato housekeeping gene ACTIN was used as an internal control. All the primers used are listed in Supplementary Table S1 and Supplementary Table S2.

\section{Histochemical GUS activity assays}

Promoters for SISWEET7a (-1486 bp) and SISWEET14 $(-1579 \mathrm{bp})$ were introduced into a pBGWES7.0 vector (including a GUS gene-coding region). The resulting fusion vector was transformed into Micro-Tom. GUS activity was examined using a GUS histochemical assay kit (Real-Time, Beijing, China) according to the manufacturer's protocol, and the tissues in which GUS was expressed were observed and imaged under a Nikon SMZ800 stereomicroscope.

\section{Generation of RNAi plants}

SlSWEET7a (Solyc08g082770) and SISWEET14 (Solyc03g097560) gene fragments of 120 and $144 \mathrm{bp}$, respectively, were amplified from tomato fruit cDNA using gene-specific primer pairs. The resulting product was cloned into a TOPO Gateway entry vector (Invitrogen, Gibco, America). The cloned fragment was subsequently transferred into a pB7GWIWG2(I) destination vector using LR Clonase II enzymes (Invitrogen, Gibco, America) according to the manufacturer's instructions. The above constructs were individually introduced into Agrobacterium tumefaciens strain GV3101. Tomato transformation was subsequently performed according to a previously described method ${ }^{57}$. The presence of transgenes in PPT-resistant plants was confirmed by PCR using a vector-specific Bar gene primer (Supplementary Table S3). Seeds obtained from the transformants were grown in growth media consisting of three parts peat, one part perlite and one part vermiculite $(\mathrm{v} / \mathrm{v} / \mathrm{v})$ in a glasshouse to obtain plants of the next generation $\left(\mathrm{T}_{1}\right)$. The $\mathrm{T}_{1}$ generation plants were subjected to screening by spraying herbicides at a concentration of $60 \mu \mathrm{g} / \mathrm{ml}$. The transgenic lines were subsequently grown to obtain nonsegregating homozygous lines. Out of 7 and 15 independent transgenic tomato lines for SISWEET7a and SISWEET14, respectively, three SISWEET7a and two SISWEET14 lines of the $T_{4}$ generation were selected and used for further analysis. 


\section{Split-ubiquitin yeast two-hybrid assays}

The coding regions (without the ATG codon and the stop codon) of SISWEET7a and SISWEET14 with SfII were amplified and cloned into a pBT3-STE bait vector, and the coding regions of SISWEET7a and SISWEET14 with SfiI were amplified and cloned into a pPR3-N prey vector. Then, the resulting vectors were transformed into yeast strain NMY51 according to the user manual (Dualsystems Biotech). Yeast transformation was performed with PEG/LiAc using a Yeastmaker Yeast Transformation System 2 Kit (Clontech, Takara, Japan). The yeast transformants were plated onto selection media lacking leucine and tryptophan but supplemented with $\beta$-galactosidase to confirm positive clones. The yeast transformants were also spread onto SD media lacking histidine, leucine, tryptophan, and adenine and supplemented with $10 \mathrm{mM}$ 3-amino-1,2,3-triazole (3-AT), which was required to prevent autoactivation of the bait vector. All the assays were repeated independently at least three times, each yielding comparable results.

\section{Bimolecular fluorescence complementation assays}

The coding sequences of the two SWEETs (without stop codons) were cloned into a Gateway pDONR221 entry vector and pXNGW (YFP) and pXCGW (CFP) Gateway destination vectors ${ }^{58}$. All the vectors were transformed into Agrobacterium tumefaciens strain GV3101. The fusion proteins were subsequently cotransformed into $N$. benthamiana leaves. Three days after transformation, the fluorescence signals were imaged using a confocal laser scanning microscope (Leica SP8, Germany). The fluorescence signals were observed at an excitation wavelength of $488 \mathrm{~nm}$ and emission wavelengths of $500-572 \mathrm{~nm}$. Each BiFC assay was repeated three times, and consistent results were obtained.

\section{Subcellular localization of SISWEET7a and SISWEET14}

The coding regions of the two SWEET genes (without stop codons) with $\mathrm{XbaI}$ and $\mathrm{KpnI}$ cleavage sites were cloned into a pCAM35-GFP expression vector. The resulting constructs were then transformed into $A$. tumefaciens strain GV3101 and used to infect $N$. benthamiana epidermal cells. mCherry-labeled AtPIP2A was used as a PM marker ${ }^{59}$. The experiments were performed independently three times. GFP fluorescence signals were observed with a confocal laser scanning microscope (Leica SP8, Germany). The fluorescence signal was observed at excitation wavelengths of $488 \mathrm{~nm}$ or $561 \mathrm{~nm}$ and emission wavelengths of $500-572 \mathrm{~nm}$ or $605-635 \mathrm{~nm}$.

\section{Complementation assays for SISWEET7a and SISWEET14 in yeast}

For complementation assays in yeast (Saccharomyces cerevisiae) cells, the ORFs of the two SWEETs with XhoI and $B a m H I$ were cloned into the yeast expression vector pDR195 ${ }^{16}$. Subsequently, the resulting constructs were transformed into the hexose transport-deficient yeast strain EBY.VW4000 and the sucrose uptake-deficient yeast strain SUSY7/ura3. Transformants of the hexose transport-deficient strain were grown on liquid SD (synthetic deficient)/-uracil media supplemented with $2 \%$ maltose (glucose was used for the sucrose uptakedeficient strain). Serial dilutions of yeast cell suspensions of EBY.VW4000 were added dropwise onto solid SD/uracil media consisting of either $2 \%$ maltose or $2 \%$ glucose/fructose. Similarly, serial dilutions of yeast cell suspensions of SUSY7/ura3 were added dropwise onto solid $\mathrm{SD} /$-uracil media consisting of $2 \%$ glucose or $2 \%$ sucrose. Growth was documented via imaging after 3-4 days of growth at $30^{\circ} \mathrm{C}$.

\section{Enzyme assays and measurements of soluble sugars and starch}

Fruits $(0.5 \mathrm{~g})$ at the MG stage were used for invertase activity analysis, as described previously ${ }^{51}$. Sucrose, glucose, and fructose were extracted in $80 \%(\mathrm{v} / \mathrm{v})$ ethanol and analyzed via high-performance liquid chromatography (HPLC), as described previously ${ }^{60}$. Starch was extracted in $9.2 \mathrm{~mol} / \mathrm{L}$ perchloric acid from the insoluble residue after soluble sugars were extracted and then filtered using filter paper $^{29}$. The supernatant and distilled water were then mixed. The starch content was determined by the anthrone colorimetric method at $625 \mathrm{~nm}$ and calculated according to a standard curve of glucose samples. Fruits $(0.5 \mathrm{~g})$ at the MG and RR stages and mature leaves $(0.2 \mathrm{~g})$ from each of the different silenced lines were analyzed for their sugar concentration as well as starch level. The soluble solid content was determined using a hand-held sugar measurement instrument (PAL-fu, ATAGO, Japan); the fruit juice of each fruit was used to obtain a reading.

\section{Statistical analysis}

The data are presented as the means \pm SDs (standard deviations). Significance tests were carried out using SPSS software (version 17.0) based on Student's $t$ tests at $P<$ 0.01 or $P<0.05$.

\section{Acknowledgements \\ We are deeply grateful to Dr. Xuan Yuan Hu from Shenyang Agricultural University for technical assistance. We especially thank Dr. Zhen Xian Zhang (China Agricultural University) for kindly providing the yeast strain SUSY7/ura3. This research was supported by the National Key Research and Development Program of China (2019YFD100030) and the National Natural Science Foundation of China (No. 31372054). We would like to thank American Journal Experts (www.aje.com) for English language editing.}

\section{Author contributions}

J.J., X.Z., C.F., T.L., and X.L. conceived and designed the experiments. X.Z., C.F., and M.W. performed the experiments. J.J., X.Z., C.F., and X.L. analyzed the data and wrote the paper. 


\section{Conflict of interest}

The authors declare no competing interests.

Supplementary information The online version contains supplementary material available at https://doi.org/10.1038/s41438-021-00624-w.

Received: 18 December 2020 Revised: 22 March 2021 Accepted: 19 April 2021

Published online: 01 August 2021

\section{References}

1. Braun, D. M., Wang, L. \& Ruan, Y. L. Understanding and manipulating sucrose phloem loading, unloading, metabolism, and signalling to enhance crop yield and food security. J. Exp. Bot. 65, 1713-1735 (2014).

2. Ruan, Y. L. Sucrose metabolism: gateway to diverse carbon use and sugar signaling. Annu. Rev. Plant Biol. 65, 33-67 (2014).

3. Li, Y., Feng, S., Ma, S., Sui, X. \& Zhang, Z. Spatiotemporal expression and substrate specificity analysis of the cucumber SWEET gene family. Front. Plant Sci. 8, 1855 (2017)

4. Zhang, C., Bian, Y., Hou, S. \& Li, X. Sugar transport played a more important role than sugar biosynthesis in fruit sugar accumulation during Chinese jujube domestication. Planta 248, 1187-1199 (2018).

5. Zhang, S. et al. Spatiotemporal transcriptome provides insights into early fruit development of tomato (Solanum lycopersicum). Sci. Rep. 6, 23173 (2016).

6. Sagor, G. H. et al. A novel strategy to produce sweeter tomato fruits with high sugar contents by fruit-specific expression of a single bZIP transcription factor gene. Plant Biotechnol. J. 14, 1116-1126 (2016).

7. Shammai, A. et al. Natural genetic variation for expression of a SWEET transporter among wild species of Solanum lycopersicum (tomato) determines the hexose composition of ripening tomato fruit. Plant J. 96, 343-357 (2018).

8. Damon, S., Hewitt, J., Nieder, M. \& Bennett, B. B. Sink metabolism in tomato fruit: II. phloem unloading and sugar uptake. Plant Physiol. 87, 731-736 (1988).

9. Osorio, S., Ruan, Y. L. \& Fernie, A. R. An update on source-to-sink carbon partitioning in tomato. Front. Plant Sci. 5, 516 (2014).

10. Wan, H., Wu, L., Yang, Y., Zhou, G. \& Ruan, Y. L. Evolution of sucrose metabolism: the dichotomy of invertases and beyond. Trends Plant Sci. 23, 163-177 (2018).

11. Julius, B. T., Leach, K. A., Tran, T. M., Mertz, R. A. \& Braun, D. M. Sugar transporters in plants: new insights and discoveries. Plant Cell Physiol. 58, 1442-1460 (2017).

12. Hackel, A. et al. Sucrose transporter LeSUT1 and LeSUT2 inhibition affects tomato fruit development in different ways. Plant J. 45, 180-192 (2006).

13. Dibley, S. J. et al. Temporal and spatial expression of hexose transporters in developing tomato (Lycopersicon esculentum) fruit. Funct. Plant Biol. 32, 777 (2005).

14. Reuscher, S. et al. The sugar transporter inventory of tomato: genome-wide identification and expression analysis. Plant Cell Physiol. 55, 1123-1141 (2014).

15. Reinders, A. et al. Protein-protein interactions between sucrose transporters of different affinities colocalized in the same enucleate sieve element. Plant Cell 14, 1567-1577 (2002).

16. McCurdy, D. W., Dibley, S., Cahyanegara, R., Martin, A. \& Patrick, J. W. Functional characterization and RNAi-mediated suppression reveals roles for hexose transporters in sugar accumulation by tomato fruit. Mol. Plant 3, 1049-1063 (2010).

17. Bologa, K. L., Fernie, A. R., Leisse, A., Loureiro, M. E. \& Geigenberger, P. A bypass of sucrose synthase leads to low internal oxygen and impaired metabolic performance in growing potato tubers. Plant Physiol. 132, 2058-2072 (2003).

18. Ayre, B. G. Membrane-transport systems for sucrose in relation to whole-plant carbon partitioning. Mol. Plant 4, 377-394 (2011).

19. Slewinski, T. L. Diverse functional roles of monosaccharide transporters and their homologs in vascular plants: a physiological perspective. Mol. Plant 4, 641-662 (2011).

20. Chen, L. Q. et al. Sucrose efflux mediated by SWEET proteins as a key step for phloem transport. Science 335, 207-211 (2012).

21. Eom, J. S. et al. SWEETs, transporters for intracellular and intercellular sugar translocation. Curr. Opin. Plant Biol. 25, 53-62 (2015).

22. Chen, L. Q. et al. Sugar transporters for intercellular exchange and nutrition of pathogens. Nature 468, 527-532 (2010).
23. Sun, $W$. et al. Cotton fiber elongation requires the transcription factor GhMYB212 to regulate sucrose transportation into expanding fibers. New Phytol. 222, 864-881 (2018).

24. Abelenda, J. A. et al. Source-sink regulation is mediated by interaction of an FT homolog with a SWEET protein in potato. Curr. Biol. 29, 1178-1186 (2019).

25. Lin, I. W. et al. Nectar secretion requires sucrose phosphate synthases and the sugar transporter SWEET9. Nature 508, 546-549 (2014).

26. Sosso, D. et al. Seed filling in domesticated maize and rice depends on SWEET-mediated hexose transport. Nat. Genet. 47, 1489-1493 (2015).

27. Yang, J., Luo, D., Yang, B., Frommer, W. B. \& Eom, J. S. SWEET11 and 15 as key players in seed filling in rice. New Phytol. 218, 604-615 (2018).

28. Chen, L. Q. et al. A cascade of sequentially expressed sucrose transporters in the seed coat and endosperm provides nutrition for the Arabidopsis embryo. Plant Cell 27, 607-619 (2015).

29. Ma, L. et al. Essential role of sugar transporter OsSWEET11 during the early stage of rice grain filling. Plant Cell Physiol. 58, 863-873 (2017).

30. Ho, L. H. et al. SISWEETla is involved in glucose import to young leaves in tomato plants. J. Exp. Bot. 70, 3241-3254 (2019).

31. Ko, H.-Y., Ho, L.-H., Neuhaus, H. E. \& Guo, W.-J. Transporter SISWEET15 unloads sucrose from phloem and seed coat for fruit and seed development in tomato. Plant Physiol. https://doi.org/10.1093/plphys/kiab290 (2021).

32. Feng, C. Y., Han, J. X., Han, X. X. \& Jiang, J. Genome-wide identification, phylogeny, and expression analysis of the SWEET gene family in tomato. Gene 573, 261-272 (2015).

33. Xuan, Y. H. et al. Functional role of oligomerization for bacterial and plant SWEET sugar transporter family. Proc. Natl Acad. Sci. USA 110, E3685-E3694 (2013).

34. Jeena, G. S., Kumar, S. \& Shukla, R. K. Structure, evolution and diverse physiological roles of SWEET sugar transporters in plants. Plant Mol. Biol. 100 351-365 (2019).

35. Le Hir, R. et al. Disruption of the sugar transporters AtSWEET11 and AtSWEET12 affects vascular development and freezing tolerance in Arabidopsis. Mol. Plant 8, 1687-1690 (2015).

36. Zhang, Z. et al. VWWWET10 mediates sugar accumulation in grapes. Genes $\mathbf{1 0}$ 255 (2019).

37. Kanno, Y. et al. AtSWEET13 and AtSWEET14 regulate gibberellin-mediated physiological processes. Nat. Commun. 7, 13245 (2016).

38. Selvam, B., Yu, Y. C., Chen, L. Q. \& Shukla, D. Molecular basis of the glucose transport mechanism in plants. ACS Cent. Sci. 5, 1085-1096 (2019).

39. Tao, Y. et al. Structure of a eukaryotic SWEET transporter in a homotrimeric complex. Nature 527, 259-263 (2015).

40. Guo, C., Li, H., Xia, X., Liu, X. \& Yang, L. Functional and evolution characterization of SWEET sugar transporters in Ananas comosus. Biochem. Biophys. Res. Commun. 496, 407-414 (2018).

41. Zhen, Q. et al. Developing gene-tagged molecular markers for evaluation of genetic association of apple SWEET genes with fruit sugar accumulation. Hortic. Res. 5, 14 (2018).

42. Jin, Y., Ni, D. A. \& Ruan, Y. L. Posttranslational elevation of cell wall invertase activity by silencing its inhibitor in tomato delays leaf senescence and increases seed weight and fruit hexose level. Plant Cell 21, 2072-2089 (2009).

43. Rottmann, T., Fritz, C., Sauer, N. \& Stadler, R. Glucose uptake via STP transporters inhibits in vitro pollen tube growth in a HEXOKINASE1-depend ent manner in arabidopsis thaliana. Plant Cell 30, 2057-2081 (2018).

44. Shen, S. et al. Cell wall invertase and sugar transporters are differentially activated in tomato styles and ovaries during pollination and fertilization. Front. Plant Sci. 10, 506 (2019).

45. Griffiths, C. A., Paul, M. J. \& Foyer, C. H. Metabolite transport and associated sugar signalling systems underpinning source/sink interactions. Biochim. Biophys. Acta 1857, 1715-1725 (2016)

46. Breia, R. et al. VVSWEET7 is a mono- and disaccharide transporter up-regulated in response to botrytis cinerea infection in grape berries. Front. Plant Sci. 10, 1753 (2020).

47. Guo, W. J. et al. SWEET17, a facilitative transporter, mediates fructose transport across the tonoplast of Arabidopsis roots and leaves. Plant Physiol. 164, 777-789 (2014).

48. Claeyssen, E. \& Rivoal, J. Isozymes of plant hexokinase: occurrence, properties and functions. Phytochemistry 68, 709-731 (2007).

49. Eveland, A. L. \& Jackson, D. P. Sugars, signalling, and plant development. J. Exp. Bot. 63, 3367-3377 (2012). 
50. Beauvoit, B. P. et al. Model-assisted analysis of sugar metabolism throughout tomato fruit development reveals enzyme and carrier properties in relation to vacuole expansion. Plant Cell 26, 3224-3242 (2014).

51. Qin, G. et al. A tomato vacuolar invertase inhibitor mediates sucrose metabolism and influences fruit ripening. Plant Physiol. 172, 1596-1611 (2016).

52. Yu, S. M., Lo, S. F. \& Ho, T. D. Source-sink communication: regulated by hormone, nutrient, and stress cross-signaling. Trends Plant Sci. 20, 844-857 (2015).

53. Sakr, S. et al. The sugar-signaling hub: overview of regulators and interaction with the hormonal and metabolic network. Int. J. Mol. Sci. 19, 2506 (2018).

54. Lastdrager, J., Hanson, J. \& Smeekens, S. Sugar signals and the control of plant growth and development. J. Exp. Bot. 65, 799-807 (2014).

55. Wang, Z. et al. Heterologous expression of the apple hexose transporter MdHT2.2 altered sugar concentration with increasing cell wall invertase activity in tomato fruit. Plant Biotechnol. J. 18, 540-552 (2019).
56. Kim, P. et al. Tissue-specific activation of DOF11 promotes rice resistance to sheath blight disease and increases grain weight via activation of SWEET14. Plant Biotechnol. J. 19, 409-411 (2021).

57. Guo, M., Zhang, Y. L., Meng, Z. J. \& Jiang, J. Optimization of factors affecting agrobacterium-mediated transformation of Micro-Tom tomatoes. Genet. Mol. Res. 11, 661-671 (2012).

58. Kim, J. G. et al. Xanthomonas T3S effector XopN suppresses PAMP-triggered immunity and interacts with a tomato atypical receptor-like Kinase and TFT1. Plant Cell 21, 1305-1323 (2009).

59. Nelson, B. K., Cai, X. \& Nebenfuhr, A. A multicolored set of in vivo organelle markers for co-localization studies in arabidopsis and other plants. Plant J. $\mathbf{5 1}$ 1126-1136 (2007).

60. Zhang, N., Shi, J., Zhao, H. \& Jiang, J. Activation of small heat shock protein (SIHSP17.7) gene by cell wall invertase inhibitor (SICIF1) gene involved in sugar metabolism in tomato. Gene 679, 90-99 (2018). 